\title{
Effect of high-fat diets on exercise performance
}

\author{
Bente Kiens* and Jørn W. Helge \\ Copenhagen Muscle Research Centre, August Krogh Institute, University of Copenhagen, 13 Universitetsparken, DK 2100 \\ Copenhagen $\emptyset$, Denmark
}

In dietary intervention studies, lasting $3-5 \mathrm{~d}$, the prevailing concept is that endurance performance after consuming a carbohydrate-rich diet is superior to that when a fat-rich diet is consumed. Thus, in the classical study by Christensen \& Hansen (1939) three trained subjects consumed either a fat-rich diet (containing only $5 \%$ energy as carbohydrate) or a carbohydrate-rich diet ( $90 \%$ energy as carbohydrate) for 3-5d. Exercise to exhaustion at approximately $65-70 \%$ maximal $\mathrm{O}_{2}$ uptake $\left(V_{\mathrm{O}_{2} \max }\right)$ revealed an average endurance time on the carbohydrate diet of $210 \mathrm{~min}$, which was markedly longer than that on the fat diet $(90 \mathrm{~min})$. Also, when intermittent exercise ( $30 \mathrm{~min}$ running followed by $10 \mathrm{~min}$ rest) at $70 \% V_{\mathrm{O}_{2} \max }$ was performed in trained men, endurance performance time to exhaustion was significantly impaired after consuming a fat-rich diet ( $76 \%$ energy as fat, $13.5 \%$ energy as protein) for $4 \mathrm{~d}$ (62 (SE 6) min) compared with a carbohydrate-rich diet $(77 \%$ energy as carbohydrate, $13.5 \%$ energy as protein) for 4d (106 (SE 5) min; Galbo et al. 1979). Also, the short-term studies by Bergström et al. (1967) and Karlsson \& Saltin (1971) suggested that 3-7d on a fat-rich diet were detrimental to exercise performance. Thus, it is evident from these short-term dietary manipulations that 'fat-loading' reduces endurance performance.

Longer-term adaptation to fat-rich diets, on the other hand, may induce skeletal-muscle adaptations, metabolic and/or morphological, which in turn could influence exercise performance. It has been known for a long time that endurance training induces several adaptations in skeletal muscle, such as increased capillarization, increased mitochondrial density, increased activity of several oxidative enzymes (Saltin \& Gollnick, 1983) and, as shown recently, an increased content of fatty acid-binding protein in the sarcolemma (Kiens et al. 1997); all variables suggested to play a significant role in enhancing lipid oxidation. It might be possible, therefore, to influence the fat oxidative system further by increasing the substrate flux of fatty acids through the system, which could be achieved by increasing the fat content of the diet. This might result in further adaptations in the fat oxidative capacity, providing possibilities for increased fat oxidation, sparing of carbohydrates and increasing endurance performance.
In the study by Lambert et al. (1994) five endurancetrained cyclists consumed, in a random order, either a diet containing $74 \%$ energy as carbohydrate or a diet with $76 \%$ energy as fat for $14 \mathrm{~d}$, separated by 2 weeks on an ad libitum or normal diet. They continued their normal training throughout the experimental period. The study revealed that maximal power output (862 (SE 94) W $v .804$ (SE 65) W for the high-fat and high-carbohydrate diets respectively) and high-intensity bicycle exercise to exhaustion at approximately $90 \% V_{\mathrm{O}_{2} \max }(8.3$ (SE 2) v. 12.5 (SE 4) min for the high-fat and high-carbohydrate diets respectively) was not impaired after the high-fat diet compared with the high-carbohydrate diet. Moreover, during a subsequent prolonged submaximal-exercise test at approximately $60 \% V_{\mathrm{O}_{2} \max }$, endurance performance was significantly enhanced on the high-fat diet compared with the high-carbohydrate diet. This improvement in submaximal endurance capacity occurred despite an initial muscle glycogen content which was twofold lower (32 (SE 6) $\mathrm{mmol} / \mathrm{kg}$ wet weight) than that in the carbohydrateadapted trial (78 (SE 5) $\mathrm{mmol} / \mathrm{kg}$ wet weight). However, the subjects performed three consecutive tests on the same day only separated by short rest intervals and the submaximal endurance test to exhaustion was always performed as the last test. This design confounds the interpretation of dietary effects on endurance performance.

In contrast, in the study by Pruett (1970) relatively welltrained subjects performed intermittent exercise tests ( $45 \mathrm{~min}$ bouts followed each time by a $15 \mathrm{~min}$ rest period) until exhaustion after consuming either a standard diet $(\%$ energy; 31 fat, 59 carbohydrate, 10 protein), a high-fat diet (\% energy; 64 fat, 26 carbohydrate, 10 protein) or a highcarbohydrate diet (\% energy; 8 fat, 82 carbohydrate, 9 protein) for at least $14 \mathrm{~d}$. Nine subjects participated in the study and each subject was placed on one of the three different diets, four of the subjects consumed all three diets. The exercise experiments were performed with 2-week intervals at workloads equal to $70 \% V_{\mathrm{O}_{2} \max }$. The subjects maintained their training throughout the 2 months required to complete the experiments. It was reported that exercise time to exhaustion was not different between the standard (175 (SE 15) min) and the high-fat diet (164 (SE 19) min),

Abbreviations: $V_{\mathrm{O}_{2} \max }$, maximal $\mathrm{O}_{2}$ uptake.

*Corresponding author: Dr B. Kiens, fax +45 3532 1567, email Bkiens@aki.ku.dk 
whereas a longer work time was observed when on the high-carbohydrate diet (193 (SE 12) min) compared with the high-fat diet (164 (SE 19) min).

In the study by Phinney et al. (1983) submaximal endurance performance was studied in five well-trained bicyclists when fed on a balanced diet for 1 week (providing 146-209 $\mathrm{kJ} / \mathrm{kg}$ per $\mathrm{d}, 1.75 \mathrm{~g}$ protein $/ \mathrm{kg}$ per $\mathrm{d}$ and the remainder of energy as two-thirds carbohydrates and one-third fat). This was followed by 4 weeks of a ketogenic diet, isoenergetic and isonitrogenous with the balanced diet, but providing less than $20 \mathrm{~g}$ carbohydrates daily. The subjects continued their normal training throughout the study. Endurance time to exhaustion, at $60-65 \% V_{\mathrm{O}_{2} \max }$, was longer in three subjects (by 57,30 and $2 \%$ ) and shorter in two subjects (by 36 and $28 \%$ ) after 4 weeks of adaptation to the ketogenic diet, resulting in no statistical difference in the mean exercise time after the two dietary trials (147 (SE 13) min for balanced diet $v .151$ (SE 25) min for ketogenic diet). However, the big variability in performance time of the subjects makes the results difficult to interpret. A highly-significant decrease in $R Q$ values during the endurance test was found and, in agreement with this finding, a threefold decrease in glucose oxidation and a fourfold reduction in muscle glycogen use were demonstrated.

The interaction between training and diet was studied by Helge et al. (1998). Fifteen initially-non-trained male subjects were randomly assigned to consume a high-fat diet (\% energy; 62 fat, 21 carbohydrate, 17 protein) or a highcarbohydrate diet (\% energy; 20 fat, 65 carbohydrate, 15 protein) while following a supervised training programme for 4 weeks. Training was performed four times weekly and each 60 min training session comprised alternating shortand long-duration periods of exercise at $60-85 \% V_{\mathrm{O}_{2} \max }$. After the 4-week intervention period $V_{\mathrm{O}_{2} \max }$ was increased by $9 \%$ in both dietary groups $(P<0.05)$. Endurance performance time to exhaustion, measured using a Krogh bicycle ergometer, at $72 \% V_{\mathrm{O}_{2} \max }$ (same absolute work load as in the initial non-trained trial), was similarly and significantly increased in both dietary groups after both 2 and 4 weeks of training and dietary treatment. Thus, comparing the trained subjects in the high-fat group with those in the high-carbohydrate group after 4 weeks, exercising at the same relative work load $\left(72 \% V_{\mathrm{O}_{2} \max }\right)$, no differences in exercise time to exhaustion were found between the two dietary groups (79 (SE 8) min high-fat group $v .79$ (SE 15) min high-carbohydrate group). Thus, it appears that adaptation to a high-fat diet in combination with training for up to 4 weeks, exercising at a submaximal workload (60-72\% $V_{\mathrm{O}_{2} \max }$ ), does not impair endurance performance (Phinney et al. 1983; Helge et al. 1998). However, in the study by Helge et al. (1996), two groups of non-trained male subjects underwent a 7-week supervised training programme while consuming either a high-fat diet (\% energy; 62 fat, 21 carbohydrate, 17 protein) or a highcarbohydrate diet (\% energy; 20 fat, 65 carbohydrate, 15 protein). $V_{\mathrm{O}_{2} \max }$ increased similarly in the two groups by $11 \%(P<0.05)$. Time to exhaustion, exercising on the Krogh bicycle ergometer at $82 \%$ pre-training $V_{\mathrm{O}_{2} \max }$, was significantly increased, from the mean initial value for the two groups of 35 (SE 4) min to 65 (SE 7) min in the high-fat

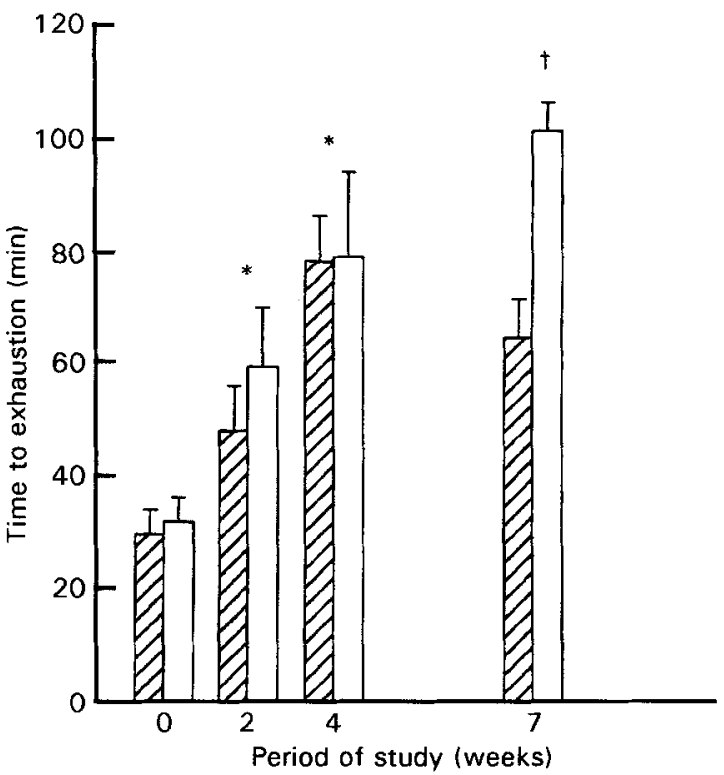

Fig. 1. Endurance performance to exhaustion measured on a Krogh bicycle ergometer before ( 0 ) and after 2, 4 and 7 weeks of endurance training when consuming a fat-rich diet $(\square)$ or a carbohydrate-rich diet $(\square)$. Mean values were significantly different from those at week 0 for both diets: ${ }^{*} P<0.05$. Mean value for the carbohydrate-rich diet was significantly different from that for the fat-rich diet at week 7 : $\dagger P<0.05$. (Data from Helge et al. 1996, 1998.)

group, with a significantly higher value in the highcarbohydrate group (102 (SE 5) min). Thus, combining these findings, it is apparent that the training-induced increase in endurance performance is less when a major part of daily energy intake consists of fat for a period longer than 4 weeks compared with when carbohydrates made up the major part of daily energy intake (Fig. 1). Furthermore, when comparing the trained subjects exercising at the same relative workload, time to exhaustion after 7 weeks on a high-fat diet was found to be significantly shorter than that with a high-carbohydrate diet. Summarizing these studies it appears that a further increase in endurance performance will be impaired when a high-fat diet is continued beyond 4 weeks. It is not clear why prolonged elevated dietary fat intake reduces improvement in endurance performance in human subjects. One important aspect of the adaptation to dietary fat could be the capacity of enzymes involved in fat oxidation; a strong correlation has been demonstrated between $\beta$-hydroxyacylCoA dehydrogenase (EC 1.1.1.35) activity and fatty acid uptake and oxidation in human subjects (Kiens, 1997). In the study by Helge \& Kiens (1997) the activity of $\beta$ hydroxyacyl-CoA dehydrogenase was increased by $25 \%$ after 7 weeks of adaptation to a fat-rich diet, irrespective of whether subjects were trained or not. Furthermore, after 4 weeks of adaptation to a fat-rich diet, carnitine palmitoyltransferase (EC 2.3.1.21) activity was increased by $35 \%$ and hexokinase (EC 2.7.1.1) activity was decreased by $46 \%$ (Fisher et al. 1983). Putman et al. (1993) demonstrated that the activity of the active form of pyruvate dehydrogenase (EC 1.2.4.1) was higher after $3 \mathrm{~d}$ of adaptation to a high-fat diet compared with a highcarbohydrate diet. Preliminary data from our laboratory 
(B Kiens, LB Turcotte and JFC Glatz, unpublished results) also reveal that a fat-rich diet per se, consumed for 4 weeks, induces a significant increase in the fatty acid-binding protein located in the cytosol and in the plasma membrane. Thus, allowing for the complexity of this issue, it seems fair to conclude that a fat-rich diet consumed for more than 4 weeks increases the capacity for fatty acid transport and oxidation. Despite this adaptation, training-induced increases in endurance performance are nevertheless impaired when compared with a high-carbohydrate diet. Thus, the fat oxidative capacity does not by itself seem to be decisive for endurance. Other explanations have to be found. Possible mechanisms could include an increased sympathetic activity with time when a fat rich diet is consumed, or changes in phospholipid-fatty acid membrane composition induced by dietary fat intake over a longer period (Helge et al. 1996). We have also demonstrated that 7 weeks of training induced a significant and similar increase in muscle insulin-sensitive glucose transporter protein GLUT4 when either a fat-rich diet or a carbohydrate-rich diet was consumed during the training period. However, during submaximal exercise at $71 \% \quad V_{\mathrm{O}_{2} \max }$, glucose uptake, measured across the thigh, was $53 \%$ lower with the fat-rich diet than with the carbohydrate-rich diet. This decrease in exercise-induced muscle glucose uptake might contribute to the impairment of endurance performance associated with the consumption of a fat-rich diet (JW Helge, EA Richter and B Kiens, unpublished results).

\section{Conclusion}

From the available literature, based on human studies, it seems fair to conclude that short-term ingestion of a fat-rich diet (3-5d) leads to a deterioration of endurance performance when compared with ingestion of a carbohydraterich diet. Moreover, adaptation to a fat-rich diet, in combination with training, from $1-4$ weeks, does not reduce endurance performance compared with a diet rich in carbohydrates, but when dietary treatment and training are continued for 7 weeks, endurance performance is markedly better when a carbohydrate-rich diet is consumed.

\section{References}

Bergstöm J, Hermansen L, Hultman E \& Saltin B (1967) Diet, muscle glycogen and physical performance. Acta Physiologica Scandinavica 71, 140-150.
Christensen EH \& Hansen O (1939) Arbeitsfähigkeit und ernärung (Physical performance and nutrition). Skandinavishes Archiv für Physiolgie 81, 160-171.

Fisher EC, Evans WJ, Phinney SD, Blackburn GL, Bistrian BR \& Young VR (1983) Changes in skeletal muscle metabolism induced by a eucaloric ketogenic diet. In Biochemistry of Exercise, vol. 13, pp. 497-501 [HG Knuttgen, JA Vogel and J Portman, editors]. Champaign, IL: Human Kinetics.

Galbo H, Holst JJ \& Christensen NJ (1979) The effect of different diets and of insulin on the hormonal response to prolonged exercise. Acta Physiologica Scandinavica 107, 19-32.

Helge JW \& Kiens B (1997) Muscle enzyme activity in man: Role of substrate availability and training. American Journal of Physiology 272, R1620-R1624.

Helge JW, Richter EA \& Kiens B (1996) Interaction of training and diet on metabolism and endurance during exercise in man. Journal of Physiology 292, 293-306.

Helge JW, Wulff B \& Kiens B (1998) Impact of a fat rich diet on endurance in man: Role of the dietary period. Medicine and Science in Sports and Exercise (In the Press).

Karlsson J \& Saltin B (1971) Diet, muscle glycogen, and endurance performance. Journal of Applied Physiology 31, 203-206.

Kiens B (1997) Effect of endurance training on fatty acid metabolism: local adaptations. Medicine and Science in Sports and Exercise 29, 640-645.

Kiens B, Kristiansen S, Jensen P, Richter EA \& Turcotte LP (1997) Membrane associated fatty acid binding protein (FABPpm) in human skeletal muscle is increased by endurance training. Biochemical and Biophysical Research Communications 231, 463-465.

Lambert EV, Speechly DP, Dennis SC \& Noakes TD (1994) Enhanced endurance in trained cyclists during moderate intensity exercise following 2 weeks adaptation to a high fat diet. European Journal of Applied Physiology 69, 287-293.

Phinney SD, Bistrian BR, Evans WJ, Gervino E \& Blackburn GL (1983) The human metabolic response to chronic ketosis without caloric restriction: Preservation of submaximal exercise capability with reduced carbohydrate oxidation. Metabolism 32, 769-776.

Pruett EDR (1970) Glucose and insulin during prolonged work stress in men living on different diets. Journal of Applied Physiology 2, 199-208.

Putman CT, Spriet LL, Hultman E, Lindinger MI, Lands LC, McKelvie RS, Cederblad G, Jones NL \& Heigenhauser GJF (1993) Pyruvate dehydrogenase activity and acetyl group accumulation during exercise after different diets. American Journal of Physiology 265, E752-E760.

Saltin B \& Gollnick P (1983) Skeletal muscle adaptability: significance for metabolism and performance. Handbook of Physiology: Skeletal Muscle, pp. 555-631 [L Peachy and $\mathrm{R}$ Adrian, editors]. Bethesda, MD: American Physiological Society. 